\title{
The Impact of Breast Reduction on Kurdish Women's Health
}

\author{
Sabir Osman Mustafa ${ }^{1}$ \\ ${ }^{1}$ Assistant Professor of Plastic and Reconstructive Surgery at Hawler Medical University and Head of Department \\ of plastic and reconstructive surgery at Rizgary Teaching Hospital/ Erbil- Iraq \\ Corresponding Author: Sabir Osman Mustafa, sabirm72@yahoo.com
}

\begin{abstract}
Background and objectives: Breast hypertrophy can cause a wide range of symptoms and affect the lifestyle and quality of life. The most common physical complaints include neck and upper back pain, shoulder pain, headache, painful shoulder grooves, low back pain, intertrigo of the inframammary crease, mastalgia, poor posture, and difficulty exercising. The cosmetic and psychological impacts of macromastia include being unable to find fitting clothes and low self-esteem. Breast reduction is the most effective treatment to improve these complaints,

Methods: This prospective study was performed on 108 patients with breast hypertrophy associated with various physical signs and symptoms that affected the quality of life and the psychological state of the patients. Reduction mammoplasty was done for these patients using a different kind of techniques. Demographic data, specific symptoms questionnaire, data from the surgical procedure, and complications were recorded and collected before the operation and three months and one year later.
\end{abstract}

Results: A hundred and eight patients with breast hypertrophy were treated surgically by reduction mammoplasty using different types of inverted T techniques from October 2008September 2019. The mean age of patients was 38.6 (13-59 years).

All patients are satisfied and described significant improvements in the symptoms and selfbody image. Postoperative complications include asymmetry (11patients), infection (2 patients), and poor scarring (6 patients).

Conclusions: Breast reduction is highly efficient in resolving symptoms and in improving health-related quality of life. It leads to a high level of satisfaction and improvement of the patient's physical and psychosocial well-being.

Keywords: breast reduction, Breast hypertrophy, patient satisfaction, reduction mammoplasty, quality of life. 


\section{INTRODUCTION}

Breast hypertrophy or macrostomia is defined as a discrepancy of breast size with body frame ${ }^{1}$, a benign, progressive enlargement of one or both breasts. ${ }^{2}$ It is a common condition that adversely affects women's physical and mental health with this disorder. ${ }^{3}$

It is responsible for collecting patients' signs and symptoms ranging from mild to devastating issues. ${ }^{4}$ The most common physical complaints include neck and upper back pain, headache, aching shoulders, painful shoulder grooves, low back pain, intertrigo of the inframammary crease, mastalgia, poor posture, difficulty exercising, and difficulty working a job without absenteeism. ${ }^{4}$

In addition to the physical dysfunctions, it significantly affects patients' social and personal lives, as it narrates self-esteem and health-related quality of life, ${ }^{4}$ which in advanced degrees may lead to impairing in daily life activities. Socially, women with breast hypertrophy try to avoid social occasions because of their feeling of being insecure regarding their bodies; therefore, 5 they tend to have more sedentary lifestyles. ${ }^{6}$ These concerns impact life quality leading to depression and anxiety problems. ${ }^{1}$

Life quality in women with breast hypertrophy is impaired, making them seek a solution to improve well-being. ${ }^{7}$

The desire for alteration in breast size is complicated and depends on many factors. ${ }^{5}$

The treatment that is quite effective for breast hypertrophy and its associated signs and symptoms is bilateral breast reduction surgery, whether vertical or horizontal methods. ${ }^{8}$ The surgery aims to decrease the breast's size and regain symmetry of chest dimensions, thus improving body image frustration. ${ }^{5}$ As it improves self-esteem, sexual function, and quality of life, decreases from anxiety depression level. ${ }^{1}$

Many surgical procedures presently exist and intend to decrease symptoms postoperatively with pleasing aesthetic outcome by restoring the breast's normal geometric shape. ${ }^{9}$

This article correlates the effect of breast reduction surgery on quality of life in patients with breast hypertrophy.

\section{PATIENTS AND METHODS}

The study took place in Erbil hospitals of the Kurdistan region between October 2008September 2019. The study sample included 108 women who had undergone bilateral reduction mammoplasty for symptomatic macromastia and met the inclusion criteria, aged 13 


\section{Proceeding of the $2^{\text {nd }}$ Scientific Conference on Women's Health 2-3 September 2020 - Hawler Medical University}

- 59 years with no previous mammary surgery history. Informed consent was obtained from all subjects.

We evaluated the quality of life, surgery outcome, and the degree of satisfaction with surgery. The patient is marked in a standing position (fig: 1). The measurements are taken. The pedicle is selected - the reduction mammoplasty technique performed under general anesthesia. The patient is in the supine position; the resected area is infiltrated with ringer lactate and adrenaline plus deepitheliazed area of the new nipple-areola position.

The procedure has consisted of the wise keyhole pattern with three different pedicles: a superomedial (62), an inferior (41), a superior pedicle (2), and a free nipple graft were used in three patients. The pillars are sutured. The nipple-areola complex is transposed to the new position. The defects are closed in layers after hemostasis, two drains are left, and lastly, dressing is done.

Patients were seen in follow up visits of a week postoperatively, then after a month and a year.

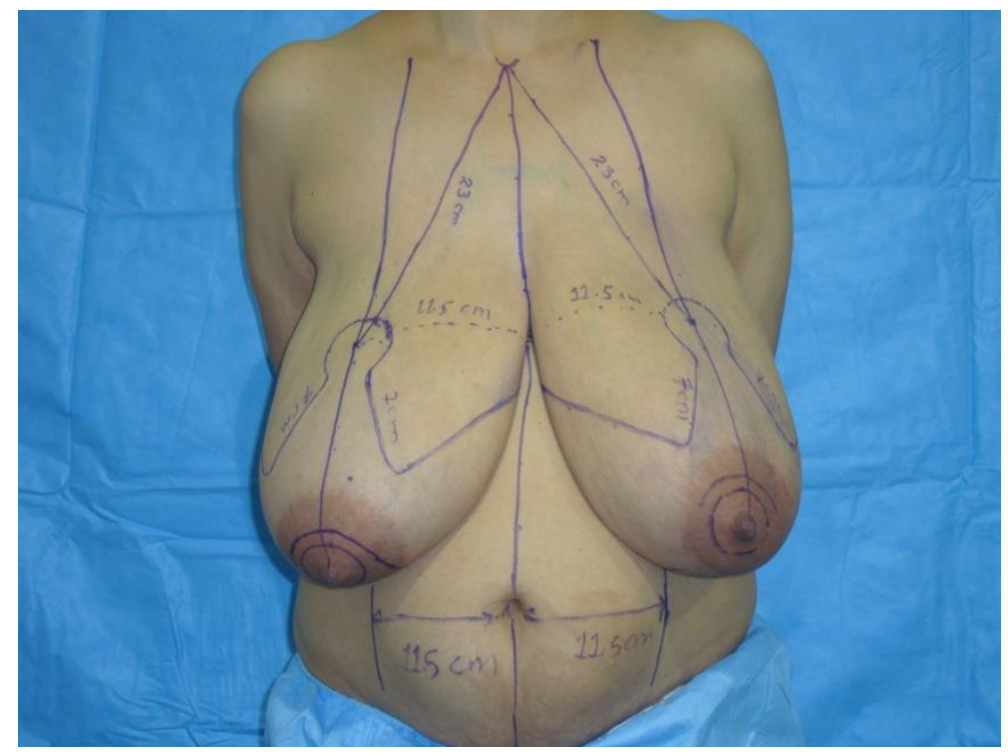

Figure 1: Marking the breasts preoperatively (inferiorly based pedicle)

\section{RESULTS}

From October 2008 through September 2019, women who underwent breast reduction surgery were 108 patients.

Overall, the mean age was 38.6 years (range $=13-59$ ); 87 of them were married, and the rest unmarried. Most of them were from urban areas. Some of them had chronic diseases. The 
majority of them were non-smokers (Table 1). Plenty of the patients had stable work (fig. 2). The combined tissue resection mass range was 1150-5300 gm.

Table 1: Demographics characteristics of patients $(\mathrm{N}=108)$

\begin{tabular}{|l|c|c|}
\hline Variables & Frequency & Percent \\
\hline Marital status & & \\
Married & 21 & 84.24 \\
Unmarried & & 22.68 \\
\hline Region & 31 & \\
Rural & 77 & 83.48 \\
Urban & & \\
\hline Past medical history & 11 & 11.88 \\
Hypertension & 4 & 4.32 \\
Diabetes mellitus & 2 & 2.16 \\
Asthma & 9 & 9.72 \\
Anemia & 3 & 3.24 \\
Bleeding disorder & & \\
Social life: & 18 & 19.44 \\
Smokers & 90 & 97.2 \\
Non-smokers & & \\
\hline
\end{tabular}

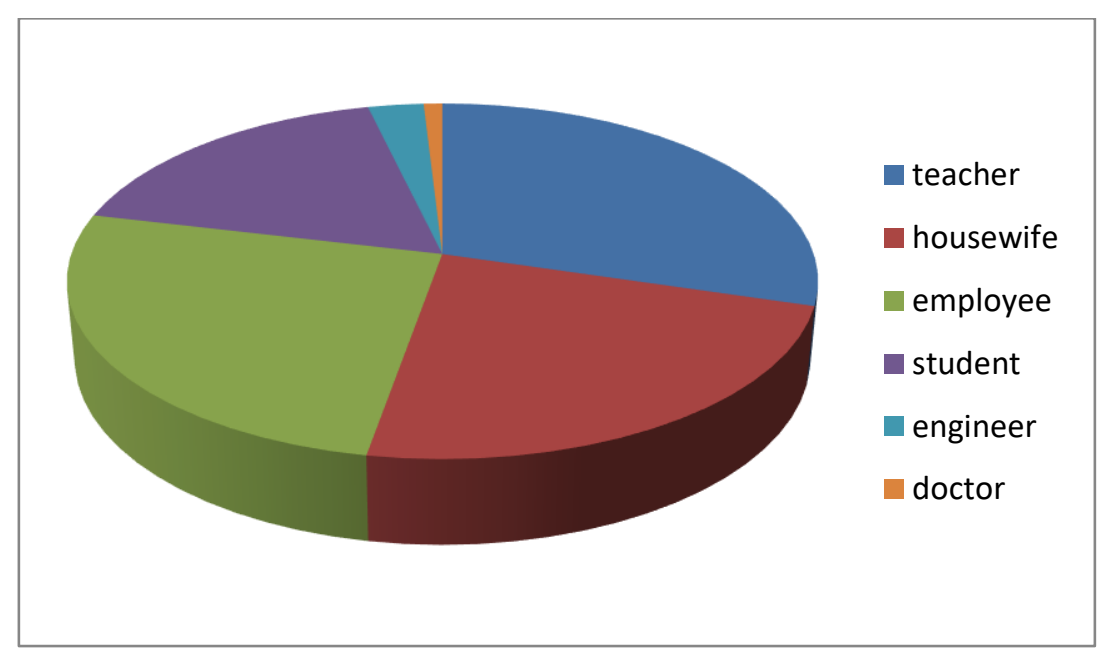

Figure 2: Occupation of the patients who underwent reduction mammoplasty 
Proceeding of the $2^{\text {nd }}$ Scientific Conference on Women's Health 2-3 September 2020 - Hawler Medical University

The study revealed that most of the patients who have undergone breast reduction surgery showed significant improvement in their quality of life and relief of symptoms. The majority of the patients were satisfied with the procedure's outcome, despite that there were complications following the surgery. The difficulties were asymmetry (11patients), infection (2 patients), poor scarring (6 patients), and altered sensation (3 patients), as shown in fig. 3.

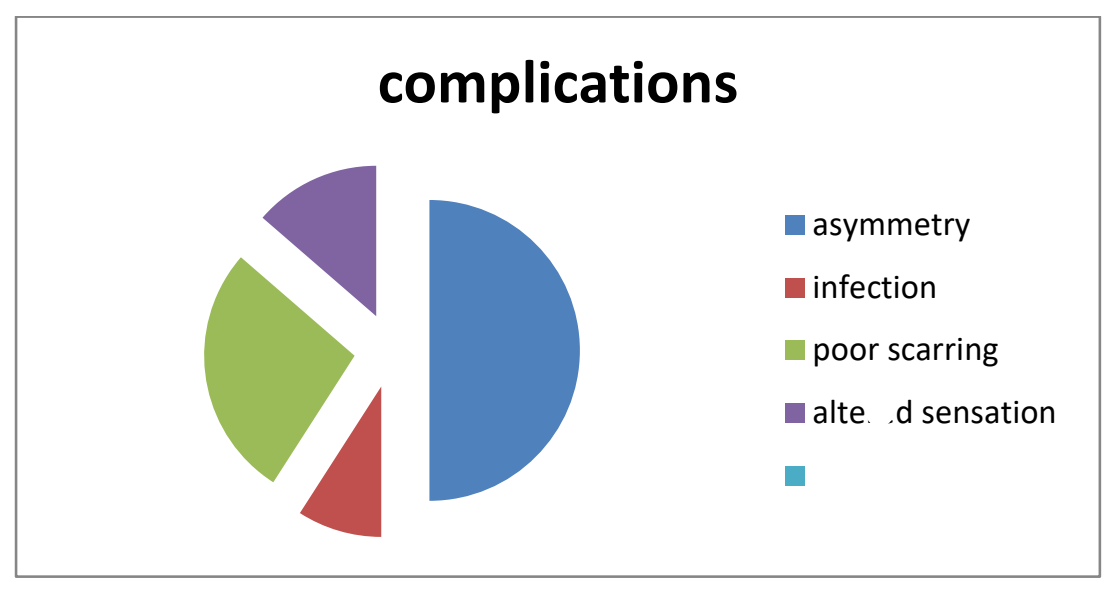

Figure 3: Complications following breast reduction surgery

There is a great change in shape and size of the breasts following reduction mammoplasty as shown in figures $4,5 \& 6$.
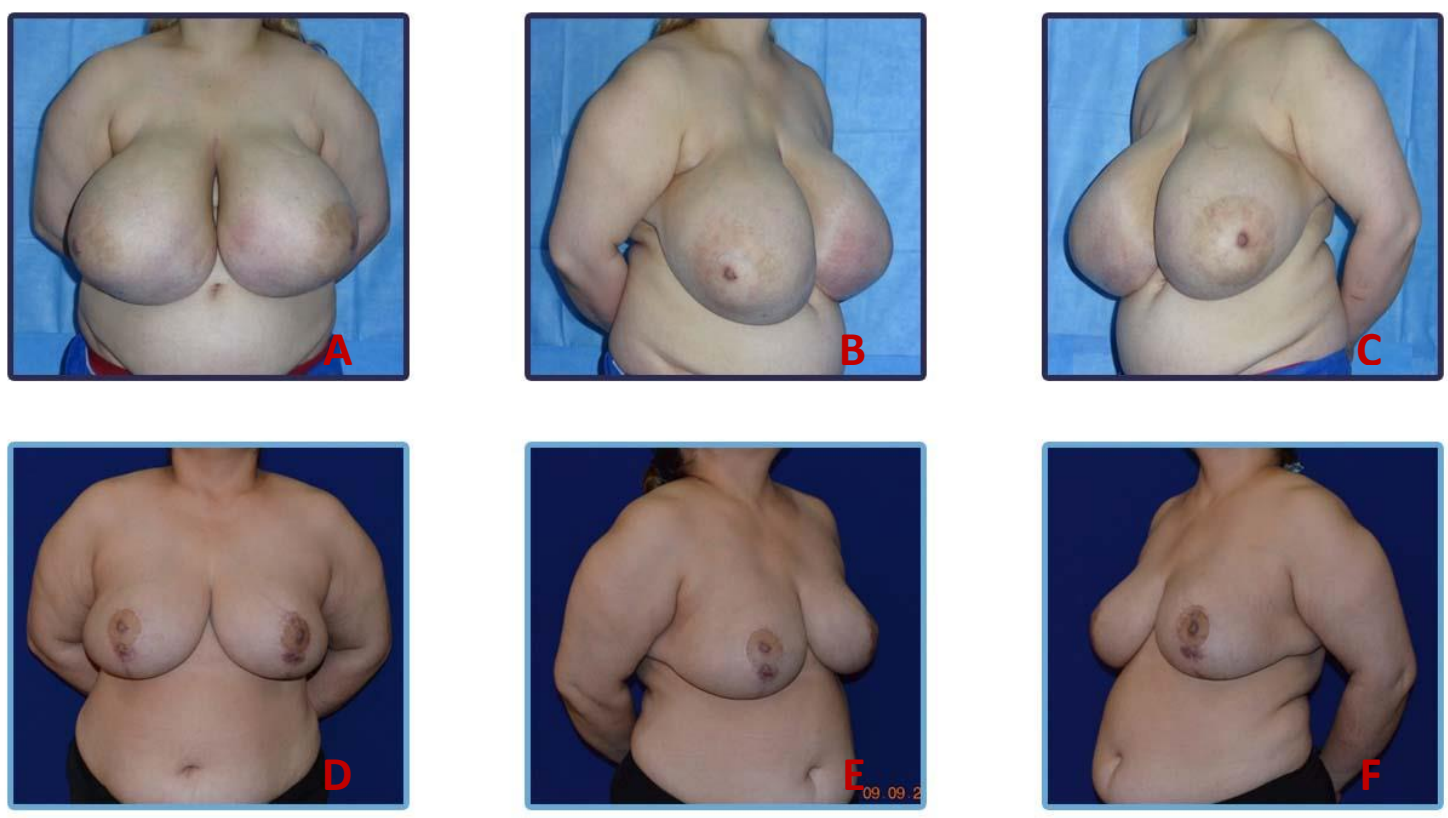

Figure: 4, showing 43-years-old lady with huge macromastia, A; anterior, B; right oblique, C; left oblique preoperative views, D; anterior, E; right oblique, F; left oblique postoperative views. 
Proceeding of the $2^{\text {nd }}$ Scientific Conference on Women's Health 2-3 September 2020 - Hawler Medical University
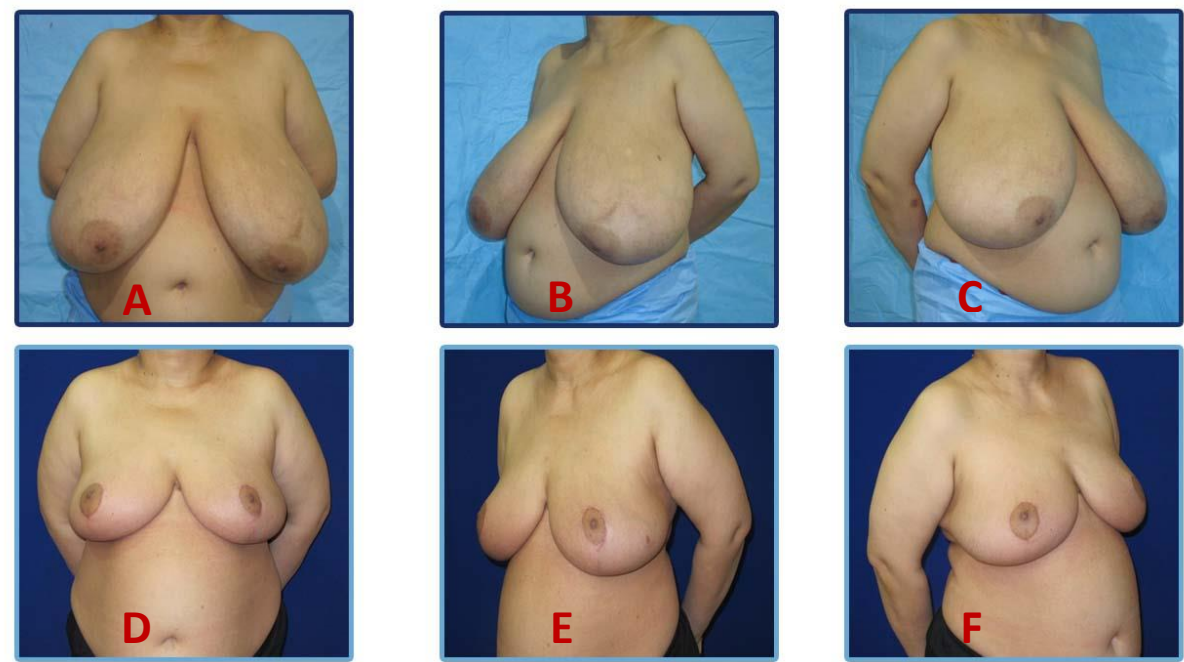

Figure. 5: A 38 year old lady with very large breasts, A; anterior, B left oblique, C; right oblique preoperative views, D; anterior, E; left oblique, F; right oblique postoperative views.
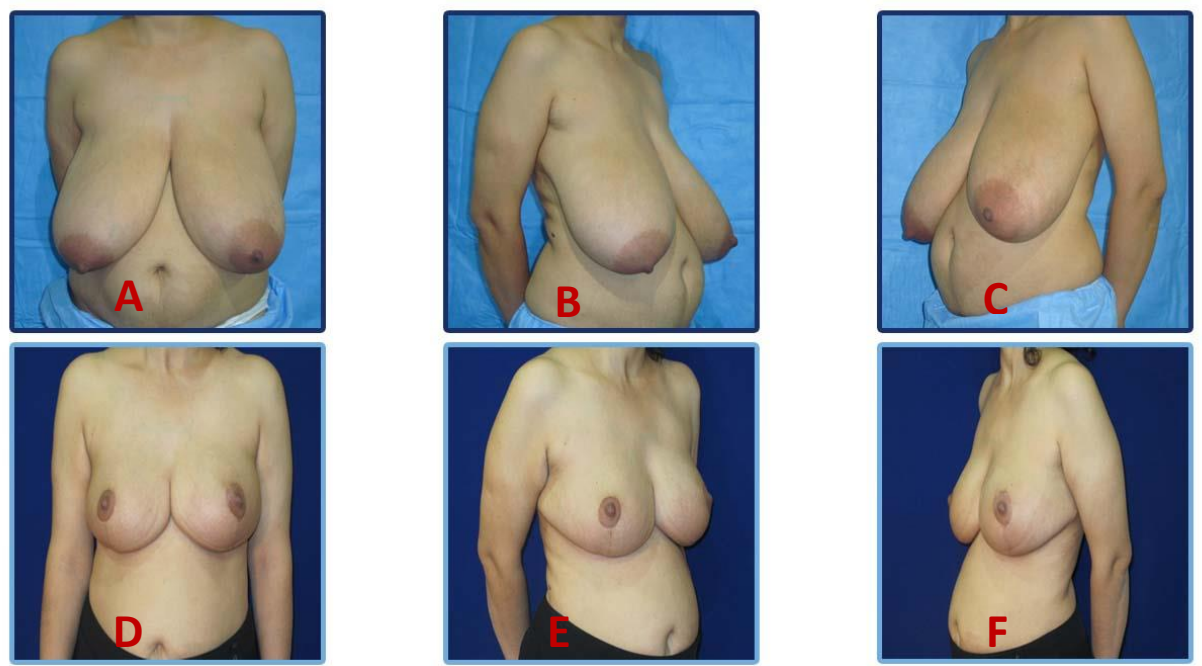

Fig: 6 shows 35-year-old lady with breast hypertrophy, A; anterior, Bright oblique, C; left oblique preoperative views, D; anterior, E; right oblique, F; went oblique postoperative views. 


\section{DISCUSSION}

In the reconstructive and aesthetic plastic surgery arena, essential components are considered for evaluating postoperative results like aesthetic parameters, complaints alleviation, and improvement in physical, social, and mental aspects of life. ${ }^{3}$

Breast hypertrophy is a clinical condition that affects many women; Lalardrie and Jouglard define it as an increase in breast volume exceeding 250-300 $\mathrm{cm}^{3}$. The disease's etiology is different: in young women, and it is mainly related to obesity, endocrine abnormalities, and virginal hypertrophy, whereas, in adult and older patients, it tends to be a mild postmenopausal effect. Large breasts can cause functional, psychological, and aesthetic discomfort, and awareness that a solution is possible has caused a dramatic increase in requests for surgical correction. $^{2}$

Reduction mammoplasty delivers great relief in physical symptoms and pain. As a result, by lessening the physical complaints caused by heavy breasts, this surgical treatment provides a tremendous improvement in life quality and physical appearance. ${ }^{10}$ It was scientifically revealed that surgery results in a significant decrease in working hours lost, impairment at work, overall productivity loss, and daily activity impairment outside of work. ${ }^{4}$ The aim of surgery to reduce mammoplasty is improvement of symptoms that patients complain of, including intertriginous infections, pain, physical inactivity, dissatisfaction with breast appearance, poor sexual well-being, and poor psychosocial well-being. All of our patients showed great relief from these problems after the surgery. Most of our patients' main complaint was the pain in the shoulders, neck, and back, which was significantly disappeared postoperatively.

The results of this study are comparable with the researches previously had been done. Our patients showed complete resolution of symptoms and significant satisfaction of $98 \%$, like that study made by Scott et al. ${ }^{12}$ on 518 patients. Physical functioning was returned to following routine surgery as that of the study made by Behmand et al. ${ }^{13}$, which was compared with nonpatient control. The patients' self-esteem and functional capacity were improved, and pain in the back region was relieved significantly following the reduction mammoplasty as Sabino Neto et al.'s study.

We found that all of our patients were more satisfied with their breast appearance, and they reported lower physical burden. Apart from this physical relief, they were more confident, selfassured, and felt more feminine and attractive after the surgery. There was also a significant 
change in body image; as a whole, women showed greater satisfaction with their bodies and positive rearrangement of the body image. The positive impact of surgery was influenced in terms of anxiety and depression, as the patients improved their quality of life. It appeared most women were pleased with aesthetic quality of their breasts postoperatively.

Younger patients $(<18$ years) showed improvement in physical well-being, vitality, psychosocial well-being, and breast-related pain and symptoms postoperatively like older patients with reduction mammoplasty surgery. The main concern in adolescent women is reoperation as a result of the growth of breasts with age. However, adult women are prone to change in morphology of breast caused by weight gain or loss, gestational breast growth, and breastfeeding. ${ }^{8}$

Generally, all of our patients were happy with their results as there was an improvement in their life compared to before the surgery. None of them regretted their decision to undergo breast reduction surgery.

\section{CONCLUSIONS}

Reduction mammaplasty significantly improves the breast-related symptoms and physical and psychosocial well-being of adolescent and young adult patients. The degree of satisfaction with the results obtained from breast reduction surgery was high, and most of the patients would recommend surgery to others and undergo surgery again.

\section{REFERENCES}

1. Pérez-Panzano E, Gascón-Catalán A, Sousa-Domínguez R, Carrera-Lasfuentes P, García-Campayo J, Güemes-Sánchez A. Reduction mammaplasty improves levels of anxiety, depression and body image satisfaction in patients with symptomatic macromastia in the short and long term. Journal of Psychosomatic Obstetrics \& Gynecology. 2017 Oct 2;38(4):268-75.

2. Cogliandro A, Barone M, Cassotta G, Tenna S, Cagli B, Persichetti P. Patient satisfaction and clinical outcomes following 414 breast reductions: application of BREAST-Q. Aesthetic plastic surgery. 2017 Apr 1;41(2):245-9.

3. Cerrato F, Webb ML, Rosen H, Nuzzi L, McCarty ER, DiVasta AD, Greene AK, Labow BI. The impact of macromastia on adolescents: a cross-sectional study. Pediatrics. 2012 Aug 1;130(2):e339-46. 
Proceeding of the $2^{\text {nd }}$ Scientific Conference on Women's Health

2-3 September 2020 - Hawler Medical University

4. Papanastasiou C, Ouellet JA, Lessard L. The Effects of Breast Reduction on Back Pain and Spine Measurements: A Systematic Review. Plastic and Reconstructive Surgery Global Open. 2019 Aug;7(8).

5. Fonseca CC, Veiga DF, Garcia ED, Cabral IV, de Carvalho MM, de Brito MJ, Ferreira LM. Breast hypertrophy, reduction mammaplasty, and body image. Aesthetic surgery journal. 2018 Aug 16;38(9):972-9.

6. Pérez-Panzano E, Güemes-Sánchez A, Gascón-Catalán A. Quality of Life Following Symptomatic Macromastia Surgery: Short-and Long-term Evaluation. The breast journal. 2016 Jul;22(4):397-406.

7. Güemes A, Pérez E, Sousa R, Gil I, Valcarreres MP, Carrera P, Gracia MD, Artigas L, Gascon A. Quality of life and alleviation of symptoms after breast reduction for macromastia in obese patients: is surgery worth it?. Aesthetic plastic surgery. 2016 Feb 1;40(1):62-70.

8. Nuzzi LC, Firriolo JM, Pike CM, Cerrato FE, Webb ML, Faulkner HR, DiVasta AD, Labow BI. The effect of reduction mammaplasty on quality of life in adolescents with macromastia. Pediatrics. 2017 Nov 1;140(5).

9. Radosa JC, Radosa MP, Baum S, Mavrova R, Camara O. Reduction mammaplasty for symptomatic macromastia: which factors influence the postoperative outcome? Archives of gynecology and obstetrics. 2013 Apr 1;287(4):715-22.

10. Hernanz F, Fidalgo M, Muñoz P, Noriega MG, Gómez-Fleitas M. Impact of reduction mammoplasty on the quality of life of obese patients suffering from symptomatic macromastia: A descriptive cohort study. Journal of Plastic, Reconstructive \& Aesthetic Surgery. 2016 Aug 1;69(8):e168-73.

11. Lonie S, Sachs R, Shen A, Hunter-Smith DJ, Rozen WM, Seifman M. A systematic review of patient reported outcome measures for women with macromastia who have undergone breast reduction surgery. Gland Surgery. 2019 Aug;8(4):431.

12. Scott GR, Carson CL, Borah GL. Maximizing outcomes in breast reduction surgery: a review of 518 consecutive patients. Plastic and reconstructive surgery. 2005 Nov 1;116(6):1633-9.

13. Behmand RA, Tang DH, Smith Jr DJ. Outcomes in breast reduction surgery. Annals of plastic surgery. 2000 Dec 1;45(6):575-80.

14. Neto MS, Demattê MF, Freire M, Garcia ÉB, Quaresma M, Ferreira LM. Self-esteem and functional capacity outcomes following reduction mammaplasty. Aesthetic surgery journal. 2008 Jul 1;28(4):417-20. 